\title{
La complétude négociée des unités de construction de tour: les complétions différées comme ressource en français parlé
}

\author{
Florence Oloff \\ ICAR, Université de Lyon, CNRS, Université Lyon 2, ENS LSH (Lyon, F-69007, France) \\ Florence.Oloff@ens-lsh.fr
}

\section{Introduction}

\subsection{Alternance des tours de parole et complétion différée}

L'alternance des tours de parole (turn-taking) entre plusieurs locuteurs en interaction est, selon l'article princeps de Sacks, Schegloff \& Jefferson (1974), fondée sur la reconnaissance de la complétude des unités de tours de parole. La complétude syntaxique, prosodique et / ou pragmatique d'une de ces unités de construction de tour (UCT) atteinte, soit le locuteur actuel peut continuer son tour en ajoutant une nouvelle UCT, soit un autre locuteur, ayant reconnu la complétude comme étant une place de transition pertinente (PTP), peut prendre la parole à son tour (Ford, Fox \& Thompson 1996a). À la base d'enregistrements d'interactions non-sollicitées et de leurs transcriptions détaillées, l'Analyse conversationnelle (pour une introduction voir ten Have 1999, Gülich \& Mondada 2001) a décrit ce principe comme étant fondamental pour l'alternance des tours de parole. La malléabilité de ces unités ainsi que la négociation située des moments de prise de parole ont été soulignées. Le locuteur actuel peut également continuer son tour au-delà de la complétude syntaxique de son tour (et donc d'une PTP) et audelà du tour d'un autre locuteur en cours, exploitant différentes suites syntaxiques possibles. Certains types de continuation de tour ("continuations d'UCT", Vorreiter 2003, "incréments", Ford, Fox \& Thompson 2002, "extensions de tour", Auer 1996) ne sont pas formatés comme étant de nouvelles unités, mais comme la suite d'une UCT ultérieure, avec un lien syntaxique et sémantique fort. L'extrait suivant (tiré de Lerner 1989: 168) illustre ce procédé:

Extrait 0

Pen: -> I don' wanna make yih ta:lk cuz I don't wantche tuh:

je n' veux pas te faire parler: parce que je ne veux pas que tu:

(.)

Pat: No: I f- I really do feel a lot [bettuh (I feel like)

Pen: -> Non: je m- je me sens vraiment beaucoup [mieux (je me sens
[upset chiself a]ll over agai:n,

[t'énerves en]core une fois:,

Si la continuation d'un premier segment (premier et deuxième segment marqués par un -> en début de ligne) s'effectue à la suite d'un tour intercalaire d'un autre locuteur (ici, le tour de Pat), nous parlons de complétion différée (CD) ("delayed completion", Lerner 1989). La complétion d'une structure syntaxique incomplète peut également être effectuée par un autre locuteur, ce qui a été traité dans la littérature sous le nom d'"énoncés construits collaborativement" (Sacks 1992), d"'énoncés-en-progrès" (Lerner 1991), de "séquences de tour collaboratives" (Lerner 2004) ou de "productions collaboratives" (Szczepek 2000). Tandis que ces derniers illustrent une co-construction syntaxique et un procédé collaboratif, la $\mathrm{CD}$ décrite par Lerner (1989) relève d'une revendication du droit de parole du premier locuteur. Une complétion différée représente un outil de gestion du chevauchement, puisque la complétion d'un tour incomplet par le même locuteur suite à un tour intercalaire sert à défendre son statut de locuteur actuel. Une CD établit un lien syntaxique étroit entre deux segments produits par le même locuteur. Même si le tour intercalaire n'a pas démarré en chevauchement, le premier locuteur exploite l'incomplétude comme ressource pour 
reprendre la parole et revendiquer son tour. La gestalt syntaxique établie à l'aide de la CD permet au premier locuteur d'identifier l'autre comme étant "hors du tour" (Lerner 1989: 171-3) et par conséquent le tour intercalaire comme interruptif.

Alors que la plupart des travaux portent sur l'anglais (pour un résumé de la littérature portant sur les incréments, voir Couper-Kuhlen \& Ono 2007), cet article donnera l'occasion d'étudier les complétions différées en français parlé et d'en identifier différentes formes. Nous allons montrer quelques-unes des multiples possibilités qu'ont les locuteurs pour établir rétrospectivement une cohérence entre deux segments "séparés" par un tour intercalaire. La première partie analytique (2.) présentera des exemples où la continuation du premier locuteur relève clairement d'un problème de droit à la parole. Pouvant apparaître après des premiers tours plus ou moins incomplets $(2.1,2.2)$, nous montrerons que la complétude d'un tour est négociée en interaction. La revendication du droit à la parole par un locuteur ne peut pas être fondée exclusivement sur des critères syntaxiques. La deuxième partie (3.) explorera le lien entre les $\mathrm{CD}$ et les complétions collaboratives. Nous présenterons des séquences avec une CD suite à un tour collaboratif d'un deuxième locuteur (3.1). Certaines constructions syntaxiques au sein du premier segment indiquent même une forte orientation vers une co-construction des tours, menant à une sorte de partage du droit à la parole (3.2). Ces séquences démontrent que la CD n'apparaît pas exclusivement dans des environnements compétitifs, mais aussi collaboratifs, dépendant également du formatage du tour intercalaire. Certaines ressources récurrentes dans différents emplacements séquentiels (tel l'usage de connecteurs) recevront une attention analytique particulière. La conclusion (4.) résumera les différents types de $\mathrm{CD}$, en insistant sur le caractère négocié des unités en interaction et le lien étroit entre grammaire et interaction. Avant de procéder à la partie analytique, nous décrirons brièvement les données utilisées.

\subsection{Description des données utilisées}

Les données analysées sont issues d'enregistrements audio et vidéo (enregistrements datant de 2004 et de 2007) d'interactions naturelles en français. Les exemples 1 à 6 sont tirés du corpus "Lyon Saxe", dont une partie est disponible sur la banque de données de corpus CLAPI (http://clapi.univ-lyon2.fr/). Il s'agit d'une conversation entre trois amis, deux hommes et une femme d'environ quarante ans, qui font une séance de travail informelle ("brainstorming") à la maison pour leur entreprise commune de marketing. Les deux derniers extraits proviennent d'un enregistrement audio effectué par nous-mêmes d'un repas de famille dans une région du nord de la France. Les cinq participants (deux hommes, trois femmes) qui se sont réunis à la maison ont entre 30 et 60 ans. Des parties de ces deux enregistrements ont ensuite été transcrites selon les conventions de transcription présentées à la fin de l'article. Ces transcriptions, en combinaison avec les enregistrements, servent de base pour les analyses suivantes. Chaque nom propre (les noms des participants ainsi que les noms et toponymes évoqués lors des conversations) a été anonymisé par un pseudonyme.

Les données utilisées pour ce travail étant de type différent (audio et vidéo), nous avons décidé de tenir compte uniquement de la piste sonore des deux corpus. Puisque divers travaux ont révélé la pertinence d'une analyse multimodale pour l'organisation de l'interaction et plus particulièrement pour l'organisation de l'alternance des tours de parole (par exemple Goodwin 1980, Mondada 2004) et puisque nous avons nous-mêmes constaté le rôle des gestes pour marquer la continuation après un chevauchement (Mondada \& Oloff à paraître), nous pouvons supposer qu'une prise en compte des ressources visibles (regards, postures, gestes etc.) pourrait contribuer considérablement à l'analyse des CD en français parlé. Lors de futurs travaux, il sera alors indispensable d'analyser également les données vidéo. Compte tenu du cadre restreint de cet article ainsi que de l'étape préliminaire des recherches sur le phénomène de la $\mathrm{CD}$ en français, il nous semblait préférable de limiter les analyses suivantes aux ressources audibles, d'autant plus qu'une analyse de l'orientation des participants vers des problèmes potentiels lors de l'alternance des tours de parole est possible à la base des détails sonores de l'interaction. 


\section{Complétions différées suite à un tour intercalaire traité comme non- collaboratif}

Une construction syntaxique incomplète dans le premier segment justifie le traitement d'un tour intercalaire comme problématique. Nous allons analyser des exemples de complétion différée $(\mathrm{CD})$ où le premier locuteur revendique, grâce à un formatage particulier de son tour, la poursuite du tour. Le traitement d'un tour intercalaire comme (plutôt) problématique peut s'effectuer suite à une incomplétude syntaxique (2.1), mais aussi après que le premier locuteur ait atteint une complétude reconnaissable (2.2).

\subsection{Complétions différées de tours "incomplets"}

Si un tour précédent n'a pas atteint une complétude syntaxique possible lorsqu'intervient un deuxième locuteur, le premier locuteur peut continuer son tour par la suite pour faire émerger une gestalt syntaxique complète. Ces cas de CD (Lerner 1989) sont également observables en français. Dans le premier exemple, Fabien (FAB) explique les problèmes dans les universités (suite à la réforme de la structuration du cursus en Licence et Master) pour trouver des stages appropriés au cursus:

Extrait 1

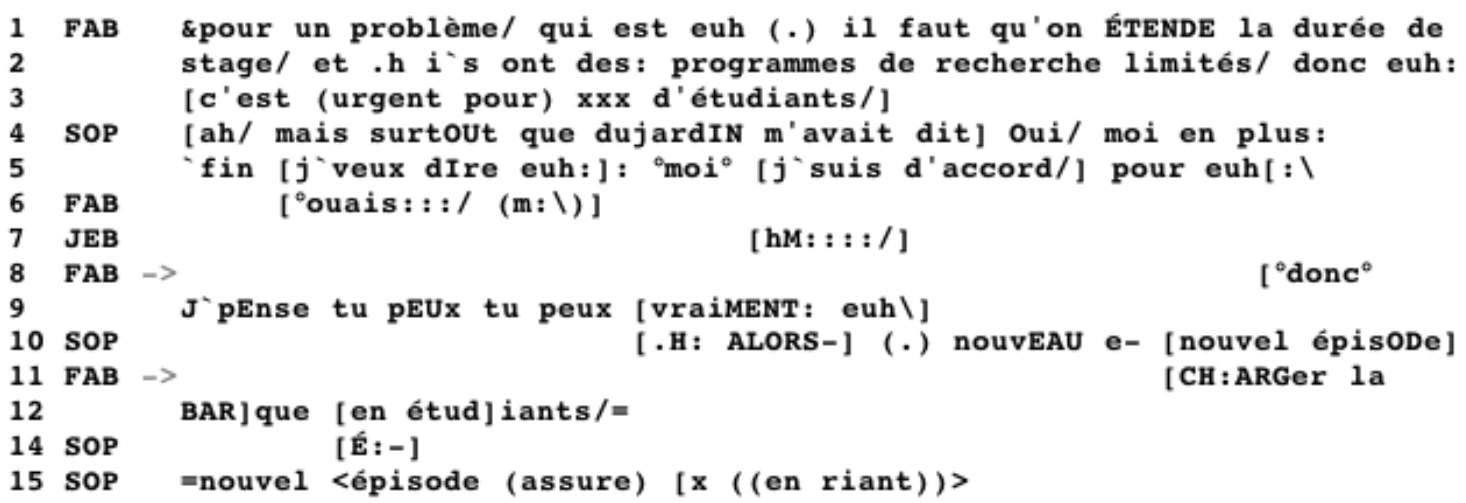

Le premier tour de Sophie (SOP, 4-5) contribue au sujet que Fabien vient de commenter, puisqu'elle évoque un entretien avec un des responsables de l'université, M. Dujardin. Puis, Fabien poursuit son raisonnement en le connectant par un "donc" à son tour précédent (8-9), mais il est chevauché de nouveau par Sophie avant que son tour n'atteigne une complétude syntaxique ou pragmatique, puisqu'il manque le complément de la construction "tu peux vraiment" (9). Cette structure syntaxique est ensuite complétée par lui (11-12) en chevauchant le tour de Sophie. L'accentuation sur "charger" et "barque" ainsi que son démarrage en chevauchement montrent le caractère potentiellement problématique de l'intervention de Sophie.

De son côté, Sophie suspend son tour pour le reprendre en clair après la fin du tour de Fabien, ce qui illustre également une orientation vers le droit de Fabien à la parole à ce moment-là. Dans ce sens, la CD est un outil du locuteur actuel (Lerner 1989: 170-1), employée pour caractériser le tour intercalaire comme interruption de l'UCT maintenant complète. Ici, cette analyse est également soutenue par rapport au nouveau topic ("nouvel épisode") que Sophie tente d'introduire avec un volume important $(10,14)$. Cette introduction visible d'un nouveau topic accentue la dimension interruptive du tour de Sophie, d'autant plus qu'il s'agit ici de la toute dernière opportunité pour Fabien de compléter son tour. Sophie reprend en répétant des bribes en et suite au chevauchement avec Fabien. L'exploitation de ces ressources par Sophie souligne également le caractère compétitif de ce tour intercalaire.

La CD telle que traitée par Lerner n'est donc pas un procédé spécifique à la langue anglaise, mais également exploité par des locuteurs du français. Néanmoins, l'(in)complétude d'un tour est plus ou moins reconnaissable et ne peut être a priori définie, puisque certains formats du tour ou certaines actions contribuent à cette difficulté de la reconnaître. Toutefois, si le format du tour même ne permet pas de 
l'établir comme visiblement incomplet à ce moment, le procédé de la CD permet au locuteur concerné d'établir ce tour rétrospectivement comme inachevé. Ainsi, dans le prochain extrait, le formatage de la fin du tour de Jean-Bertrand (JEB) comme le début d'une liste peut indiquer une possible incomplétude, mais c'est seulement grâce à la $\mathrm{CD}$ ensuite que le premier tour est traité par lui comme étant incomplet. Dans cet exemple, Jean-Bertrand parle d'une stratégie à adopter envers un client potentiel qui vend des produits à base de céréales. La liste que Jean-Bertrand commence vers la fin de son premier tour - bien que cette liste ne soit pas complète en elle-même - n'a qu'une faible force projective, comme le montre son formatage sonore. En effet, Jean-Bertrand continue ensuite cette structure syntaxique possiblement complète en chevauchement avec Sophie:

Extrait 2

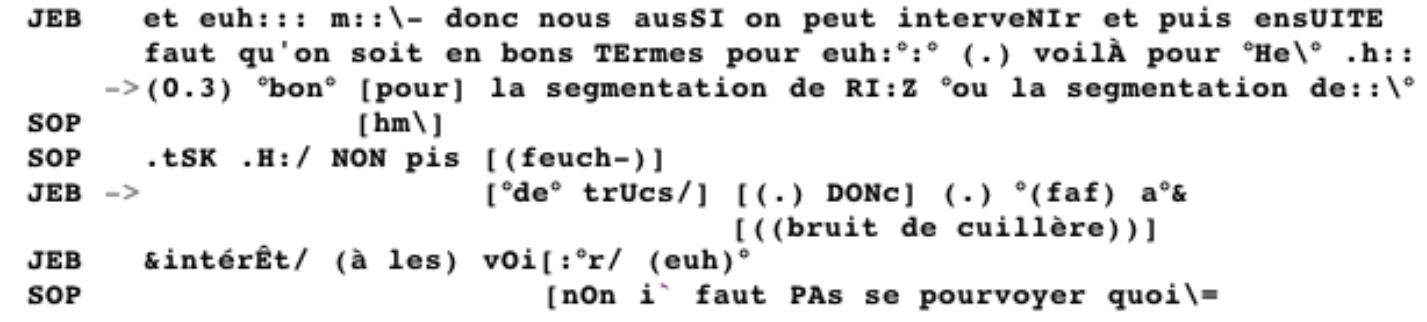

Au début de cet extrait, Jean-Bertrand évoque un client potentiel pour leur entreprise de marketing et la stratégie à adopter (1-3). D'un côté, la fin de son tour projette syntaxiquement une suite, le deuxième complément ("pour la segmentation de riz" "ou la segmentation de" --> x) n'ayant pas encore été produit. De l'autre, son tour montre non seulement de nombreuses hésitations ("euh", "voilà", "bon" et double répétition de "pour"), indiquant d'éventuels problèmes pour trouver la suite appropriée du tour, mais aussi une diminution du volume, un allongement vocalique sur le dernier mot ("de::") ainsi qu'une intonation descendante. Ces phénomènes indiquent plutôt une fin de sa prise de parole. De plus, le fait qu'un premier complément ait déjà été donné ("de riz", 3) diminue la nécessité d'un deuxième complément.

Sophie entame un nouveau tour à cet endroit (5), mais cette UCT est chevauchée par Jean-Bertrand avant une place de transition pertinente (PTP) (6). Il énonce le deuxième complément "manquant" de son premier tour ("de trucs") avant de produire une suite (introduite par "donc") portant probablement sur une personne dont le surnom est "faf". Bien que la continuation de la première partie du tour de Jean-Bertrand n'apporte rien de nouveau au niveau informationnel (utilisation du pro-terme "truc"), elle clôt la gestalt syntaxique. Ainsi, Jean-Bertrand traite son premier tour (1-3) rétrospectivement comme incomplet. Puisque la continuation n'apporte rien de nouveau d'un point de vue sémantique, la CD est ici exclusivement exploitée pour la revendication du tour de Jean-Bertrand.

Il est intéressant de noter que Sophie, bien que commençant hors chevauchement, semble s'orienter vers une négociation éventuellement problématique du droit à la parole. Ceci se manifeste dans le volume de sa voix relativement augmenté ainsi que dans le bruit de succion et d'aspiration très audibles, marquant clairement le début d'un nouveau tour (5). Cette orientation témoigne probablement de sa difficulté à interpréter le tour précédent de Jean-Bertrand comme explicitement complet ou non. Mais l'abandon de son tour dès les premières syllabes en chevauchement montrent aussi qu'elle traite son propre droit à la parole comme étant à ce moment plus faible que celui de Jean-Bertrand.

Ces doubles orientations des participants montrent que la complétude d'un tour peut être établie rétrospectivement et qu'elle est négociée tour par tour par les participants. Bien qu'il soit possible de juger la complétude ou non d'une construction syntaxique à partir de critères de la grammaire standard, l'exemple 2 illustre qu'une structure incomplète à ce niveau peut être construite comme ne projetant pas de suite, grâce à la modulation de la voix ou à l'aide d'allongements vocaliques et de bribes répétées. L'incursion de Sophie dans le "tour" de Jean-Bertrand ne peut donc pas être constatée à partir d'une structure syntaxique complète ou non du premier tour, mais uniquement à partir de leurs propres orientations dynamiques, manifestes dans la façon de construire leurs tours respectifs par la suite. Les 
deux prochains exemples montreront également que la complétude d'une unité en interaction est négociée par les participants plutôt que d'être un fait grammatical (cf. aussi Mondada à paraître).

\subsection{Complétions différées de tours "complets"}

Différentes formes d'extension de tour ("add-on", "remplacement", "incrément", "glue-on" etc., cf. Vorreiter 2003) peuvent être exploitées comme ressource pour construire la continuation. Cette variété de suites possibles souligne que la poursuite d'un tour ne peut pas être mise en relation automatique avec une incomplétude du segment précèdent ( $\mathrm{cf}$. extrait 2). Au contraire, bien qu'une complétude syntaxique puisse être clairement atteinte lors d'un premier tour, le locuteur peut construire sa prochaine prise de parole comme la suite de son tour précédent. Si cette deuxième partie est syntaxiquement indépendante, l'établissement rétrospectif d'une incomplétude s'effectue souvent à l'aide de connecteurs ou de particules discursives, particules qui apparaissent parfois de façon cumulée. Dans l'extrait suivant, Jean-Bertrand donne l'exemple d'un producteur régional appartenant aux concurrents d'un client potentiel:

\section{Extrait 3}

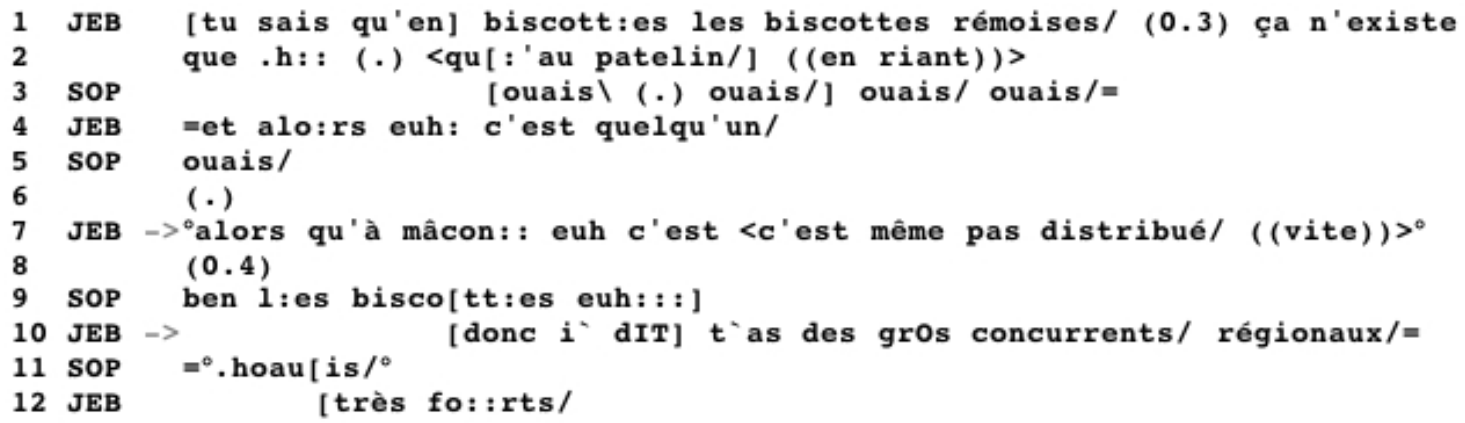

Jean-Bertrand donne l'exemple d'un producteur de biscottes qui, bien que ses produits ne soient pas distribués dans d'autres régions, est bien positionné sur le marché de sa région, ce que Sophie accompagne par des continuateurs répétés (1-6). A la fin de la ligne 7, Jean-Bertrand arrive à un point de complétude pragmatique (le "alors" indiquant l'opposition entre la position forte régionale d'un côté et "l'absence" de présence au niveau national de l'autre) et syntaxique (l'intonation montante en fin de tour n'étant pas une intonation projective, marquant plutôt le lien logique entre les deux parties (4 \& 7), les deux étant inversées: "alors que ce n'est pas distribué à Mâcon, le producteur a quand même une position forte en région champenoise"). De plus, nous pouvons observer une diminution du volume accompagnée d'une accélération vers la fin. La pause ensuite de 0.4 secondes (8) ainsi que la prise de parole de Sophie nous indiquent la présence d'une PTP (9).

Pourtant, Jean-Bertrand chevauche le tour de Sophie - bien avant une complétude de celui-ci - constituant la pause avant comme n'étant pas une occasion de prise de parole pour elle (10). Bien que possiblement indépendant au niveau syntaxique ("il dit t'as des gros concurrents", 10), Jean-Bertrand exploite le "donc" pour établir un lien avec son UCT précédente. Même si le formatage de ce tour n'illustre pas une compétition pour le tour en particulier (pas d'augmentation du volume ou de la vitesse, pas de recyclage ou de répétitions de mots), l'abandon ("drop out", cf. Schegloff 2000) presque immédiat de Sophie et le continuateur suivant (11) démontrent son orientation vers un droit fort au tour de Jean-Bertrand.

Dans le quatrième extrait, un procédé similaire est exploité lors de la discussion autour des fluctuations de produits dans les grandes surfaces (cf. aussi l'extrait 5): 


\section{Extrait 4}

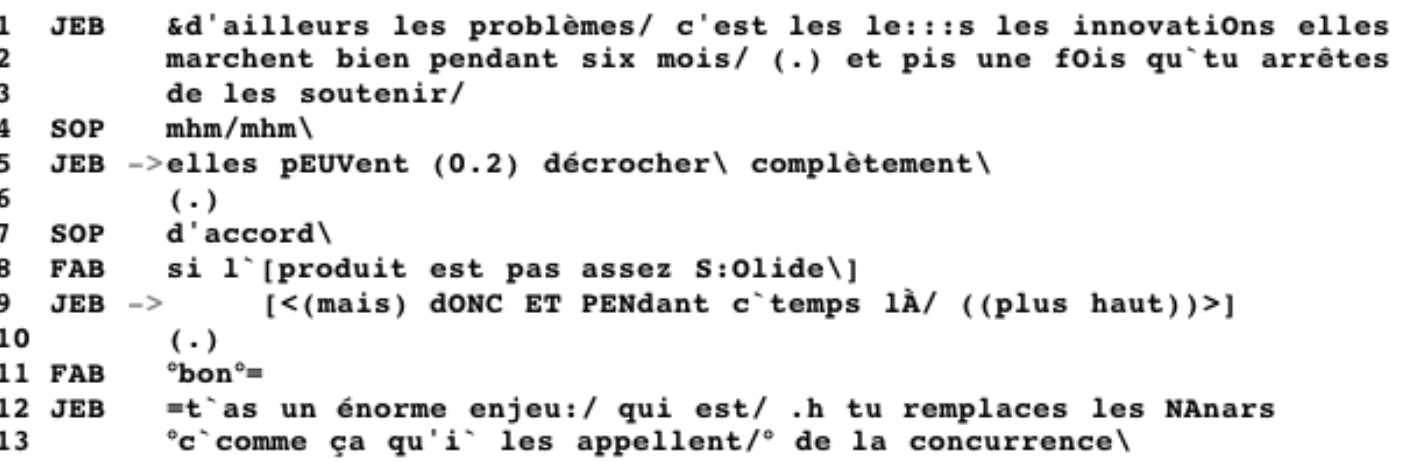

L'explication de Jean-Bertrand sur la longueur de vie de produits novateurs atteint une fin à la 1. 5. La structure syntaxique est visiblement complète, les derniers mots recevant une emphase particulière et la prosodie étant descendante, conclusive. Tandis que Sophie n'émet qu'un continuateur après la micropause (7), Fabien saisit cette place de transition pertinente (PTP) pour énoncer un jugement sur la qualité de ces produits (8). De manière intéressante, ce tour représente aussi une continuation possible de la conclusion de Jean-Bertrand (5). Mais cet énoncé potentiellement collaboratif n'est pas validé en tant que tel: après à peine une syllabe, le tour de Fabien est chevauché par Jean-Bertrand (9) qui, jusqu'à la fin du chevauchement, augmente le ton de sa voix et met une emphase particulière sur ses mots, traitant l'intervention de Fabien comme compétitive.

Suite au chevauchement (12), Jean-Bertrand continue sa construction syntaxique débutée en chevauchement (9). Il est intéressant de constater qu'au niveau informationnel, cette continuation représente plutôt une ouverture sur un nouveau cadre temporel et représente une sorte d'introduction projetant une suite ("pendant ce temps là", 9). En contraste avec cette valeur projective et prospective, les connecteurs en début de la CD ("mais donc et", 9) établissent un lien rétrospectif. Vu l'emplacement en chevauchement à la suite d'un premier segment du même locuteur (1-3,5), ces connecteurs cumulés (9) ne semblent pas correspondre à des marqueurs de début de tour, mais signalent une continuation du segment précédent. Malgré une complétude (aussi bien syntaxique que prosodique et pragmatique) du premier segment, Jean-Bertrand revendique ici le tour à l'aide de la CD.

L'utilisation des connecteurs dans les deux exemples précédents est en lien avec leur fonction en interaction - la création d'une incomplétude d'un tour ultérieur au-delà d'un point de complétude reconnaissable et reconnu par les interlocuteurs. Situés dans ce que Auer (1996) appelle le pre-front field d'une UCT, les connecteurs semblent (dans le deuxième segment d'un même locuteur, souvent en chevauchement) indiquer l'orientation du participant vers son statut de locuteur actuel. Elles n'établissent pas de véritable lien sémantico-syntaxique (de conséquence, de conclusion etc.), fonction attribuée aux connecteurs dans le cadre d'une grammaire standard. Ceci relève probablement de la grammaticalisation de certains connecteurs du français qui, dans des environnements séquentiels précis, prennent une valeur de marqueur discursif (pour une étude de la grammaticalisation de quelques connecteurs en allemand, voir Auer \& Günthner 2003, voir aussi Fox 2007 qui décrit le positionnement récurrent de certains éléments dans un tour ou au sein d'une séquence comme un procédé possible de grammaticalisation). Dans les extraits 1 (8) et 2 (6), nous pouvons également constater l'emploi de connecteurs dans cette position séquentielle. Si d'autres séquences de CD démontrent une exploitation systématique de certaines particules discursives en français parlé (notamment "donc"), il serait peut-être possible de les définir en tant qu"'indicateurs" de continuité (au-delà d'un point de complétude).

Le fonctionnement des connecteurs comme outil de gestion de l'alternance des tours de parole semble être lié à leur emplacement précis en position initiale du tour dans une séquence, plus précisément en début de tour chevauchant suite à une prise de parole du même locuteur juste avant (donc au sein d'une CD). Tandis que les particules discursives sont des traces visibles dans la transcription et donc faciles à repérer, 
il faudrait, dans de futurs travaux, également étudier leur formatage phonétique précis. Ainsi, Local \& Kelly (2006) illustrent la prolongation d'un tour au-delà d'une pause à l'aide du coup de glotte en anglais parlé. Ceci souligne que la projection (cf. Auer 2002) d'une suite plus ou moins immédiate au premier segment n'est pas analysable uniquement à base de la syntaxe, mais que d'autres ressources (dont la modulation des sons) interviennent lors de l'émergence d'une (in)complétude. La complétude d'un tour n'est pas un fait indiscutable ou préexistant à l'interaction, mais le résultat d'une négociation entre les participants.

\section{Complétions différées suite à des complétions collaboratives}

Il est remarquable que les complétions différées semblent être fréquemment précédées de tours intercalaires collaboratifs (Lerner 1989: 173-175). L'extrait 4 a illustré l'exemple d'un tour intercalaire potentiellement collaboratif qui, grâce à un formatage particulier de la $\mathrm{CD}$, a été traité comme concurrentiel par le premier locuteur. Dans les extraits suivants (présentant des cas clairs de complétions collaboratives), ces tours collaboratifs sont traités (par le locuteur qui effectue la CD) comme plutôt nonproblématiques. En effet, la complétion du premier segment par la suite peut témoigner de ce procédé collaboratif en position intercalaire en ayant un format moins compétitif que dans les exemples précédents. Les prochains paragraphes vont explorer en détail différents cas de co-occurrence entre complétion collaborative et complétion différée. La reprise du tour intercalaire par le premier locuteur n'est pas a priori analysable comme revendiquant le tour, mais tout d'abord comme reçu (positif) de la complétion collaborative (Lerner 1989: 174). Mais accompagné de certaines caractéristiques de formatage de tour, ce reçu peut simultanément être construit comme une CD.

\subsection{Complétions collaboratives après une incomplétude du premier tour}

Bien que des cas de CD puissent témoigner de l'orientation des participants vers une prise de parole intercalaire problématique, ce procédé n'est pas exclusivement traité comme relevant d'un problème de prise de parole, voir d'une interruption du locuteur actuel. L'incomplétude d'un segment reconnue comme telle par un autre locuteur peut également être l'occasion de le compléter. L'extrait suivant illustre comment un tour intercalaire peut être traité par le premier locuteur comme collaboratif (à un certain moment), tout en revendiquant son tour par une CD:

\section{Extrait 5}

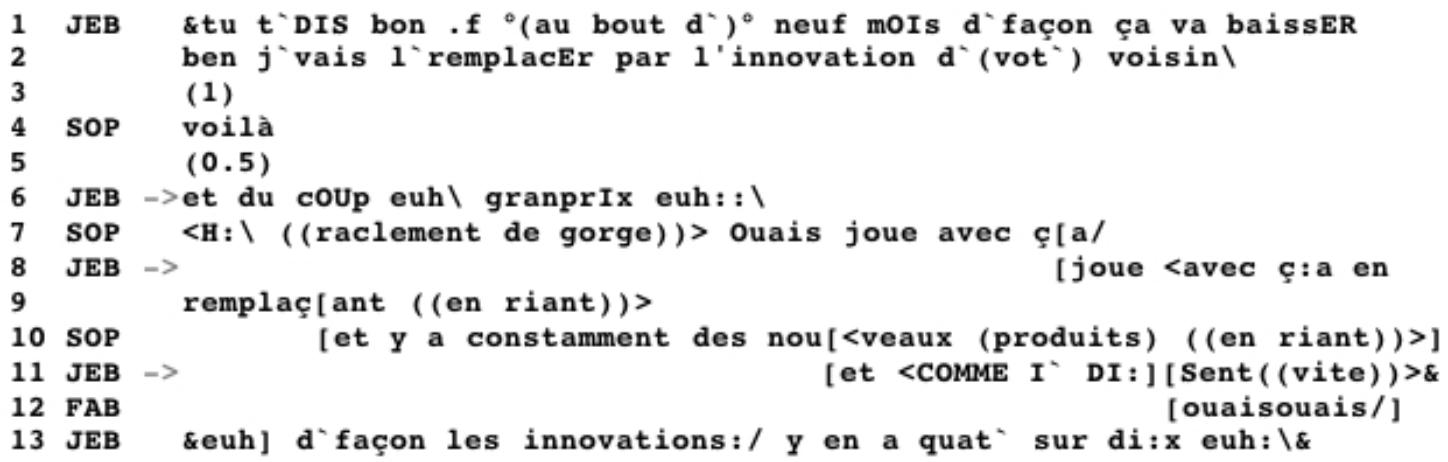

Jean-Bertrand vient de terminer une séquence d'explication sur une stratégie d'innovation de produits dans les entreprises productrices et la fluctuation des produits dans les grandes surfaces (cf. ex. 4). Son début de conclusion (6) par rapport à la politique des grandes surfaces (dont "grandprix" est une enseigne) est complété par Sophie (7). Sophie s'oriente ici vers le caractère inachevé du tour de Jean-Bertrand, qui démontre en effet une certaine difficulté de continuer le tour à ce moment (hésitation et allongement, 6). Ensuite, Jean-Bertrand émet un reçu positif de la proposition de Sophie en la répétant littéralement, et en complétant son premier segment de son côté. Le fait qu'il chevauche à peine le tour de Sophie, le volume 
de la voix non-modifié par rapport à son premier tour aussi bien que le rire montrent qu'à cet endroit, le tour intercalaire n'est pas traité comme problématique.

Que ce segment soit possiblement le début d'une CD revendiquant le tour (et non un simple reçu de la complétion collaborative) est visible par la suite: après avoir validé l'intervention de Sophie, mais en même temps regagné le tour, Jean-Bertrand s'oriente vers son droit de locuteur actuel (11). Il chevauche Sophie pour continuer avec un autre segment (qui, par le connecteur "et", est relié aux UCT précédentes et donc construit comme la suite). Le volume augmenté de sa voix et la légère accélération désignent ici un démarrage de tour compétitif (French \& Local 1986). A cet emplacement, Jean-Bertrand traite le deuxième tour de complétion collaborative de Sophie (10) comme plutôt problématique, ce qui indique que la cession du droit à la parole à un autre locuteur est négociée moment par moment. Très probablement, l'acceptation ou non d'une complétion collaborative dépend également de son degré d'adaptation syntaxico-sémantique au tour inachevé: tandis que le premier tour de Sophie est une complétion nécessaire du premier segment et intervient suite à des difficultés exhibées par Jean-Bertrand d'achever le tour, le deuxième n'intervient pas à la suite d'un problème dans le tour de Jean-Bertrand, de plus, il est syntaxiquement moins intégré (relié par le "et"). Ce deuxième tour de Sophie représente plutôt une sorte de conclusion, à laquelle Jean-Bertrand semble opposer sa propre suite. De manière intéressante, il emploie ici le même connecteur que Sophie ("et", cf. 10 \& 11), ce qui pourrait témoigner d'un éventuel alignement (quoique minimal) avec Sophie. Mais comme Jean-Bertrand débute son tour au milieu de l'UCT en cours de Sophie, il semble le concevoir plutôt comme une version alternative, en compétition avec la suite proposée par Sophie.

Ceci illustre que la CD n'est pas automatiquement l'indicateur d'une prise de parole problématique d'un autre locuteur. L'emplacement de la CD par rapport au tour intercalaire (en chevauchement avant sa complétude ou plutôt vers sa fin / une PTP) ainsi que son formatage (volume, débit etc.) permettent au premier locuteur d'exploiter ce procédé pour revendiquer son tour de façon plus ou moins marquée. Un autre type intéressant de CD non-marquée est illustré par l'extrait suivant, dans lequel Fabien accepte la complétion collaborative de Sophie tout en l'insérant dans sa propre complétion de tour. Au début de l'exemple, Fabien est en train de parler du cursus professionnel d'un certain Jérôme ou Philippe (dont le nom exact n'est pas connu des participants), qui depuis peu est le PDG d'une des entreprises détenues par son père, M. Bougetron:

\section{Extrait 6}

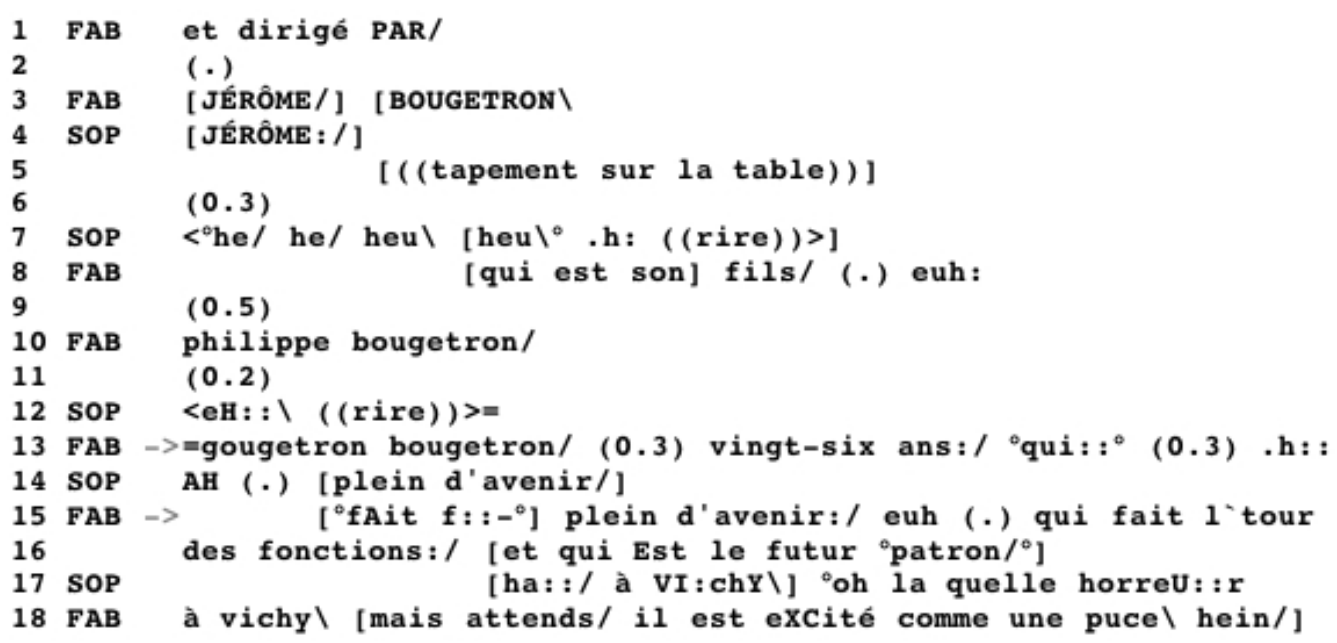

Dans cet extrait, Fabien évoque une personne qui travaille dans l'entreprise paternelle, dont ni le prénom, ni le nom de famille sont connus avec certitude de Fabien (ce qui explique l'utilisation de deux prénoms différents pour la même personne et la présence du rire). Bien que Fabien soit en train de construire un tour complexe (en donnant des informations sur cette personne peu à peu), les interventions de Sophie sont traitées par Fabien comme étant non-problématiques puisqu'elles sont courtes et interviennent à des 
moments précis, témoignant de son alignement avec lui. Fabien suspend pour une micropause son tour (2), occasion qui est saisie par Sophie pour compléter simultanément avec Fabien cette UCT (3-4). Suite au traitement de cette blague (Sophie et Fabien ayant fait un jeu de mots sur le nom juste avant, le vrai nom leur étant inconnu, cf. aussi le début de la 1. 13), Fabien revient à la description de la personne et poursuit avec plus d'informations sur elle $(8,10,13)$. Après avoir donné son âge, Fabien projette visiblement une suite (introduite par "qui" et s'intégrant dans la liste des informations sur la personne), mais suspend son tour (13). Sophie, qui s'oriente vers cette suspension comme étant un problème de recherche de suite de tour, propose la description "plein d'avenir" en chevauchement avec Fabien (14). Au lieu de continuer sa construction syntaxique entamée en chevauchement ("fait f::- ${ }^{\circ}$, 15), Fabien va d'abord accepter cette proposition en la répétant avant de continuer sa construction syntaxique ultérieure (15-16).

Bien que Fabien effectue ici une CD, il traite le tour de Sophie non comme une incursion, mais comme une intervention collaborative qui peut être intégrée dans la suite qu'il projetait. De manière intéressante, Sophie construit son tour en reprenant la construction appositive ("vingt-six ans:/", 13) introduite par Fabien et non la construction utilisant le pronom relatif ("oqui::", 13), en ajoutant une descriptions en relation étroite avec la dernière bribe d'information disponible dans le tour de Fabien ("vingt-six ans", cette personne est jeune et donc "plein d'avenir"). Aussi bien au niveau du contenu qu'au niveau syntaxique, Sophie crée un lien plutôt rétrospectif que prospectif. Elle ne propose donc pas une suite alternative - et par conséquent concurrentielle - au segment inachevé de Fabien, mais une sorte de recyclage, ce qui souligne le caractère collaboratif de son intervention. Dans cet extrait, la CD indique la fin de la résolution collaborative d'un problème (proche d'une recherche de mots), et nous voyons que cette complétion ne contient pas d'éléments susceptibles d'indiquer une prise de parole problématique de la part de Sophie, le volume ainsi que le débit du tour de Fabien étant non-modifiés. Bien que le démarrage en chevauchement de la $\mathrm{CD}$ puisse être traité comme un premier essai de Fabien de ne pas tenir compte de la proposition de Sophie et d'éliminer ainsi sa pertinence (cf. Lerner 1989: 175), la suspension de la complétion de Fabien pour une prise en compte explicite de la proposition "plein d'avenir" fait que le tour de Sophie est rétrospectivement traité comme pertinent. L'insertion d'un reçu positif (par un acquiescement explicite ou une reprise) dans la CD permet aux participants de tenir simultanément compte de deux complétudes possibles sans pour autant perdre le droit au tour.

Si une complétion collaborative est proposée au premier locuteur suite à une incomplétude de son tour, plusieurs enchaînements à celle-ci sont possibles en CD: premièrement, la refuser explicitement (par un "non" par exemple) ou la refuser en continuant sa $C D$ en chevauchement (Ex. 5: 11), deuxièmement, ni la refuser, ni l'accepter clairement en continuant directement après la complétude du tour intercalaire (pour un exemple voir Szczepek 2000: 6), ou, troisièmement, l'accepter en l'insérant avant ou pendant la poursuite de la construction initiale (ex. 5: 8-9 \& 6: 15). Les cas des recherches de mots ou de "suites de construction" représentent une particularité dans le sens où les hésitations et / ou la suspension momentanée d'une construction syntaxique favorisent des complétions collaboratives de la part des autres locuteurs.

Le degré de problématicité du tour intercalaire dépend non seulement de son lien avec le premier (nouvelle projection, cf. ex. 1-3, ou complétion collaborative, ex. 5-6), mais aussi d'éventuelles attentes que le premier segment, malgré son incomplétude, peut projeter envers un autre locuteur. Ainsi, une hésitation à la fin d'une construction incomplète peut être reconnue par un autre locuteur comme signalant un problème de poursuivre ce tour à ce moment-là (dû à une recherche de mots). Des problèmes manifestés dans la construction de la suite du premier segment peuvent en effet laisser attendre une complétion collaborative de la part d'un interlocuteur. Il existe alors des formatages de tour qui peuvent en quelque sorte déclencher un certain type de tour intercalaire. Ainsi, le début d'une relative ("qui", ex. 6: 8 \& 13) ou la construction de liste ("vingt-six ans", ex. 6: 13; ici une liste des caractéristiques d'une personne) représentent des structures syntaxiques particulièrement favorables à la complétion collaborative. Les derniers extraits montreront un autre type de construction, des structures binaires, qui semblent projeter une complétion collaborative en même temps qu'une suite. 


\subsection{Suspension de construction et projection de complétion collaborative: partager le tour}

Même si un premier locuteur projette clairement une suite (ce qui est par exemple le cas dans des activités qui impliquent des tours complexes, comme les récits), un deuxième locuteur peut parfois enchaîner par une complétion collaborative qui est non seulement traitée comme non-problématique, mais aussi attendue par le premier locuteur. Les prochains extraits proviennent d'une conversation entre deux demisœurs, Noëlle et Suzanne, qui, lors d'un apéritif en famille, passent en revue des éléments de leur jeunesse commune.

\section{Extrait 7}

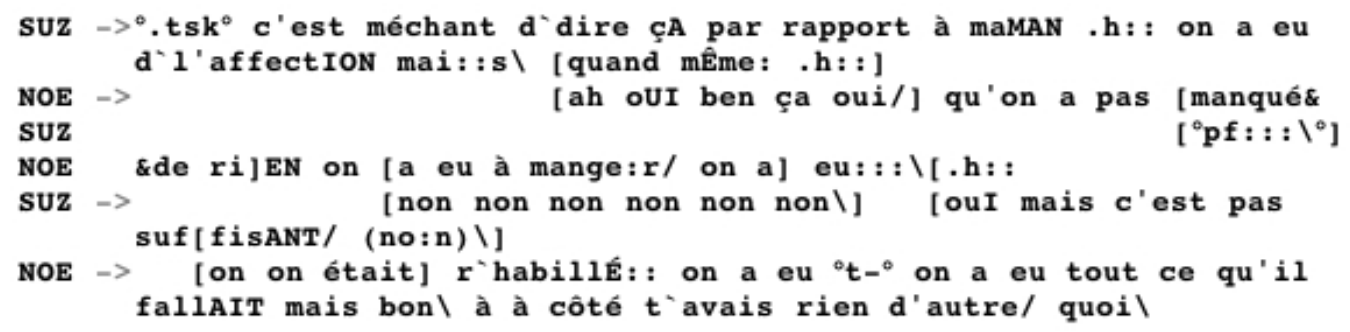

Le premier tour de Suzanne projette comme suite une structure oppositionnelle ("on a eu x, mais", 1-2). En la chevauchant, Noëlle acquiesce d'abord pour ensuite ajouter des éléments à la première partie, faisant une sorte de liste dont le premier élément était fourni par Suzanne ("on a eu de l'affection, on a manqué de rien, on a eu à manger" etc., $3 \& 5$ ). De son côté, Suzanne acquiesce d'abord en répétant cinq fois un "non" (6), tenant ainsi compte de la construction en négation de Noëlle. C'est seulement lorsque Noëlle termine sa liste avec un allongement vocalique (5) qu'elle fournit une deuxième partie pour sa construction oppositionnelle ("mais c'est pas suffisant", 6-7), sans pour autant s'orienter vers un caractère potentiellement problématique de la prise de parole de Noëlle. Au contraire, elle valide ce tour par son "oui" (6).

Il est vrai que le "quand même" (2) pourrait être analysé comme la complétion de la structure binaire (d'opposition) de Suzanne, ce qui rendrait la complétion plus tard obsolète. Mais nous pouvons constater que la "deuxième" complétion (6-7) propose, contrairement à la première, une véritable conclusion au niveau informationnel. De plus, l'aspiration de Suzanne en chevauchement (2) projette une suite au-delà du "quand même", terme qui peut donc être analysé comme ouvrant sur un deuxième élément ou une suite oppositifs. La deuxième complétion (6-7) semble même avoir été alimentée par le tour intercalaire de Noëlle. Ici, l'alternance des tours de parole prend la forme d'un récit collaboratif entre les deux sœurs, par opposition à un récit classique avec une distribution de la parole plus asymétrique. Cette activité collaborative se caractérise par des sortes de suspensions d'un tour suivies par des tours intercalaires collaboratifs, qui à leur tour peuvent ensuite être complétés, ce qui efface la frontière nette entre les complétions différées et collaboratives. Des séquences collaboratives similaires apparaissent par exemple lors de descriptions co-construites (Mondada 1999). De manière intéressante, nous pouvons observer une continuation de la liste de Noëlle dans le dernier tour de l'extrait (8-9), qui se termine par une opposition similaire à celle de Suzanne, introduit par un "mais". Éventuellement, des ressources visibles pourraient être déployées systématiquement à un tel endroit de façon à projeter aussi bien audiblement que visiblement une forme collaborative d'un deuxième tour (cf. Bolden 2003), piste à explorer dans des futurs travaux portant sur la multimodalité de la CD en français parlé. Dans ce type d'activité, il semble exister une autre forme de $\mathrm{CD}$, qui, d'un côté, laisse un créneau reconnaissable pour des interventions collaboratives, et qui, de l'autre, exploite le matériel de ces contributions collaboratives. Le dernier extrait illustre des interventions collaboratives quasiment déclenchées par un tour précédant: 
Extrait 8

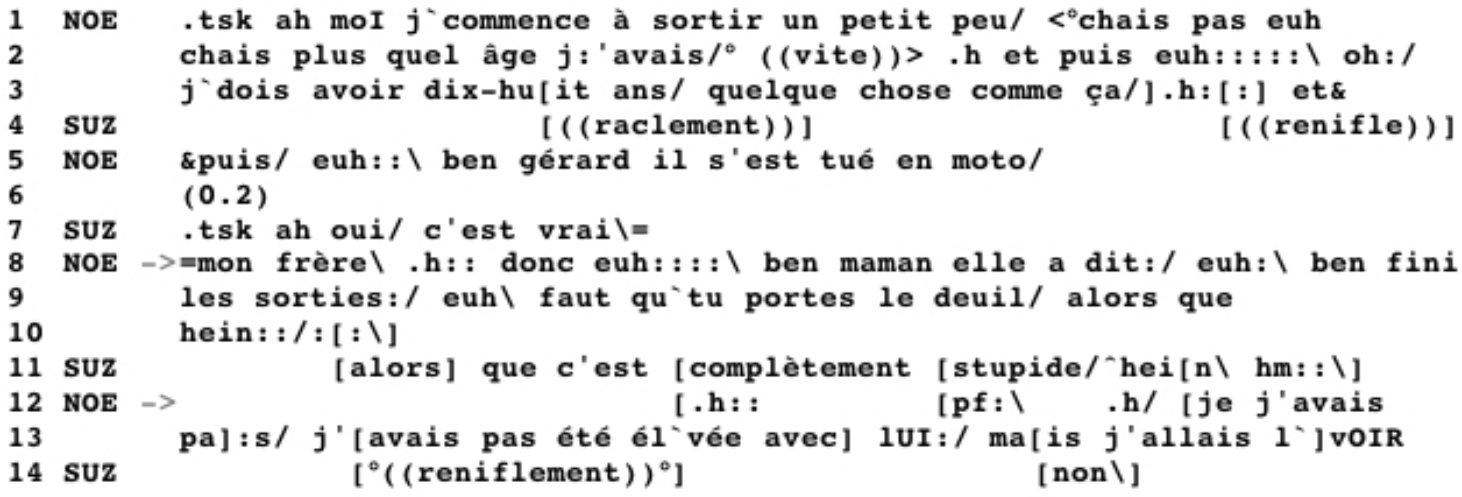

Noëlle explique ici qu'elle n'avait plus le droit de sortir le soir à partir du moment où son frère (qui avait grandi dans un autre foyer qu'elle et Suzanne) avait eu un accident mortel de la route (1-5, 8-9). En citant leur mère (chez qui les deux demi-sœurs avaient grandi ensemble), Noëlle projette ensuite une construction contrastive par un "alors que" (9). Mais elle ne la développe pas tout de suite, terminant son tour par un "hein" allongé (10), pendant lequel Suzanne entame une complétion collaborative en exploitant le même connecteur (11). Tandis que le "alors que" projette clairement une suite du premier tour de Noëlle, le "hein" suspend la construction syntaxique momentanément. Ici, le "hein" de Noëlle ne correspond pas à un marqueur de fin de tour, mais plutôt à une demande d'évaluation ("assessment", Pomerantz 1984) du discours de leur mère. L'allongement du "hein" ainsi que l'intonation montante et ensuite descendante créent une véritable place de transition possible. De manière intéressante, cette particule est reflétée dans le "hein" à la fin de la complétion évaluative et complétive de Suzanne (11), de façon à ce que cette contribution paraisse être la forme complète d'une première construction elliptique de Noëlle. Le fait que ce deuxième "hein" ne fonctionne pas comme déclencheur d'une évaluation est visible dans sa modulation, énoncé sans allongement et collé à "stupide" avec une intonation descendante.

Le "pf:" (en tant qu'évaluation négative du discours de leur mère, 12) de Noëlle représente une sorte de première complétion du segment au niveau de son contenu, tout en manifestant un accord avec l'évaluation négative de Suzanne. Ceci permet à Noëlle de prendre la parole en chevauchement avec Suzanne sans qu'il n'y ait une "lutte pour le tour" (Schegloff 2000). Le recyclage (Schegloff 1987) de la première partie de la suite de Noëlle ("j'avais pas", 12-13) comme manière de sortir du tour démontre même son orientation vers le droit de Suzanne au tour à ce moment-là. Cette construction de Noëlle pourrait être analysée comme une structure syntaxique indépendante, il semble alors difficile de distinguer une nouvelle UCT d'une continuation (CD). Néanmoins, la brièveté entre la fin du "hein" (10) et l'aspiration (12) de Noëlle ( 0.2 secondes) démontrent plutôt une continuité de ce tour respectivement sa suspension (ressemblant à un "gearing up", cf. Jefferson 1984). Cette continuité est également reflétée au niveau des droits, qui se manifestent dans le formatage des tours: tandis que Suzanne complète de son côté par un "c'est" impersonnel (11), Noëlle a recours à la première personne du singulier pour construire ses tours. Ceci positionne Noëlle comme la source principale de la narration, Suzanne ne la prenant pas en charge de la même façon.

Ce dernier exemple montre la possibilité de "suspendre" une structure incomplète (incomplète puisqu'une suite a été projetée) afin de projeter des contributions spécifiques d'un autre locuteur telles des évaluations ou des acquiescements complétants. Qu'une structure syntaxique quelconque puisse être traitée comme "momentanément complète" et ensuite rétrospectivement comme incomplète est également illustré au début du même extrait: le segment "gérard il s'est tué en moto" (5) est syntaxiquement complet, et l'intonation montante non-interrogative. Cette intonation ne projette non seulement une suite (le lien entre la mort de son frère et ses sorties), mais laisse également une place (pause de 0.2 secondes, 6) pour un acquiescement de Suzanne. Il s'agit d'une possibilité d'établir cet événement comme relevant d'un savoir partagé. Tout de suite après que Suzanne se soit souvenue de façon reconnaissable de cet événement 
(indiqué par le change-of-state-token "ah", cf. Heritage 1984), Noëlle continue en enchaînement rapide par un "remplacement" (Vorreiter 2003) ("(gérard) / (mon frère) s'est tué en moto", 8). Elle établit ainsi une incomplétude particulière de son tour précédent. La CD permet donc l'insertion de séquences d'évaluation tout en ne mettant pas en négociation le droit à la parole d'un locuteur. En combinaison avec des complétions collaboratives, les CD permettent une co-construction d'une suite de tours entre deux ou plusieurs locuteurs. Si une complétion indique une défense du tour ou bien son partage dépend alors fortement du type d'activité et de séquence en cours (cf. Fox, Ford \& Thompson 1996b), autant que le formatage des tours de ces séquences (formatés comme plus ou moins compétitifs, plus ou moins collaboratifs) nous renseigne sur le genre d'activité en cours.

\section{Conclusion}

Les extraits présentés visaient à analyser des cas de complétions différées $(\mathrm{CD})$ en français parlé. Tandis que Lerner (1989) insiste notamment sur leur fonction de défense du tour et d'effacement de pertinence d'un tour intercalaire, nos analyses ont également présenté des cas témoignant de procédés collaboratifs. Bien que la $\mathrm{CD}$ en français semble être un moyen de revendiquer son droit à la parole, sa présence n'équivaut pas automatiquement à des tours intercalaires problématiques. Il convient de remarquer que le terme d'interruption utilisé par Lerner (1989: 171-3) pour décrire le phénomène des CD ne représente pas une véritable catégorie descriptive (pour une discussion cf. Schegloff 2002): l'interruption convient à un ensemble limité de cas de CD (comme l'exemple 9 dans Lerner 1989: 172), il semble alors logique que ce terme ne devrait pas être mis en relation exclusive ou automatique avec le procédé de la CD. Nous préférons par conséquent décrire différentes formes de $\mathrm{CD}$ comme plus ou moins compétitives ou problématiques, ou bien comme plus ou moins collaboratives (cf. point 3.2). Le tableau ci-dessous illustre ces deux possibilités distribuées sur un continuum (en forme de flèche), avec des formes de CD plutôt problématiques, concurrentielles à gauche et des formes plutôt non-problématiques, collaboratives à droite. 


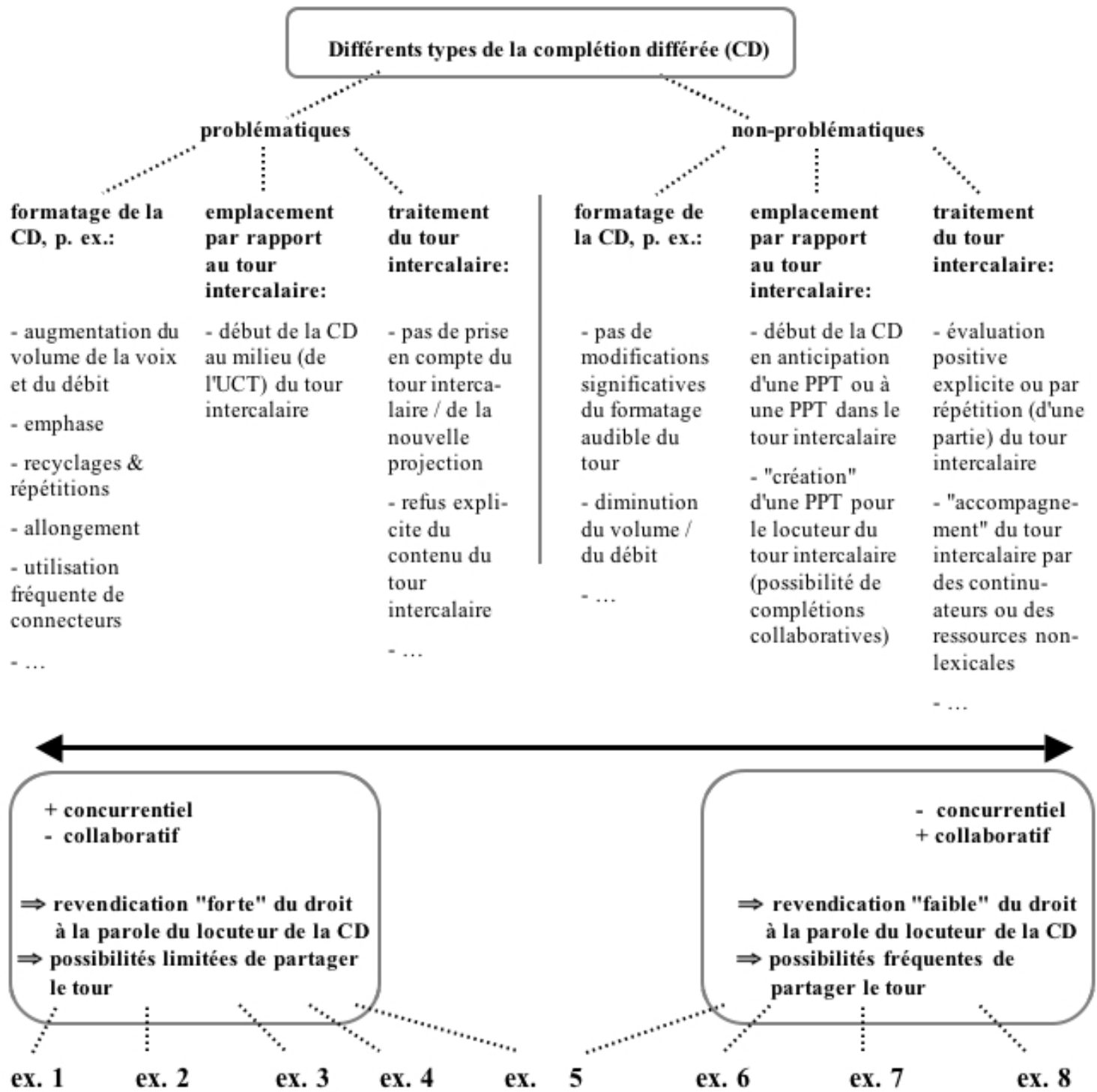

Les analyses précédentes visaient à démontrer l'exploitation variée de ce procédé, variations qui se manifestent à plusieurs niveaux: premièrement, au niveau du formatage de la CD (ici, nous avons tenu compte notamment du formatage sonore), deuxièmement, au niveau de l'emplacement de la $\mathrm{CD}$ - son début - par rapport au tour intercalaire et, troisièmement, au niveau de la manière dont est traité le tour intercalaire. Le tableau de conclusion montre ces trois points dont nous avons tenus compte lors des analyses précédentes en haut, avec des regroupements de phénomènes témoignant d'une CD plutôt problématique (à gauche) ou d'une CD plutôt non-problématique (à droite). Ces listes de phénomènes sont ouvertes. En outre, ceux-ci peuvent soit apparaître de façon cumulée (notamment pour le formatage de la CD), soit représenter différentes possibilités pouvant être sélectionnées par les locuteurs. Tout en bas du tableau figurent les extraits traités dans cet article, attribués à un type de CD plutôt concurrentiel (extrait 1 à 4), relevant d'une revendication forte du droit à la parole, ou bien à une CD plutôt collaborative (extrait 6 à 8), avec une revendication plus faible du droit à la parole ainsi qu'une possibilité augmentée de partage de tour. L'exemple 5 peut être partiellement attribué aux cas problématiques, 
partiellement aux cas non-problématiques, l'analyse ayant démontré une première CD collaborative avec une $\mathrm{CD}$ concurrentielle à sa suite.

Les participants peuvent faire varier le formatage d'une CD de sorte à ce que le tour intercalaire soit traité comme problématique ou non. Ainsi, la modulation de la voix lors de la complétion peut témoigner d'une compétition, correspondant à une reprise de tour marquée suite à un chevauchement ("marked turn retrieval", Jefferson 2004). Ceci se caractérise par une augmentation du volume de la voix, une emphase particulière ou une accélération (French \& Local 1986) (notamment ex. 1, 2 et 4). Mais la complétion ne peut s'accompagner d'aucune de ces caractéristiques ou alors présenter même une diminution du volume ou des recyclages, ce qui en fait des complétions moins marquées par rapport à la revendication du tour. Si la CD intègre du matériel du tour intercalaire (ex. $5 \& 6$ ), elle s'oriente vers des complétions moins compétitives, s'accompagnant souvent de problèmes tels une recherche de mots. D'autres CD semblent même apparaître après des projections d'un certain type de tour intercalaire (évaluation ou complétion collaborative, ex. $7 \& 8$ ). Ces cas démontrent la continuité d'une prise de parole avec une possibilité simultanée de prise de parole d'un autre participant. Le traitement du tour intercalaire comme plutôt problématique ou collaboratif dépend alors fortement du formatage de celui-ci, mais aussi de l'emplacement de la CD par rapport au tour intercalaire. Nous pouvons remarquer que dans les exemples 1-4 (5), les locuteurs démarrent leur CD bien avant une place de transition pertinente dans le tour intercalaire, souvent au milieu de l'UCT en cours. Une orientation vers un partage de tour ou une revendication plus faible du droit à la parole se manifeste au contraire dans une CD débutant en anticipant légèrement ou en attendant une PPT dans le tour intercalaire, comme dans les exemples (5) 6-8.

Si l'on s'intéresse au formatage des tours intercalaires, nous pouvons constater que dans le cas d'un formatage d'une CD comme "problématique", le tour intercalaire semble plus fréquemment correspondre à une nouvelle projection, qu'il traite d'un autre topic (ex. $1 \& 2$ ) ou du même (ex. $3 \& 4$ ). Le formatage d'une CD comme non-problématique ou même collaborative semble par contre apparaître dans des séquences où le tour intercalaire correspond à une complétion collaborative (ex. 5 \& 6) ou à une évaluation complétive qui s'intègre dans l'activité en cours (ex. $7 \& 8$ ).

Par conséquent, le critère de l'(in)complétude syntaxique du premier segment ne permet pas à lui seul de prévoir le type de formatage de la $\mathrm{CD}$ à suivre. Au lieu de pouvoir être rattachée uniquement à des critères syntaxiques, la complétude d'une unité en interaction est soumise à une négociation entre les participants, dépendant des activités en cours et de leur gestion de l'alternance des tours de parole (Selting 2000). Les extraits présentés ont montré qu'une CD peut suivre sur différents types d'(in)complétude, l'incomplétude d'un tour pouvant être établie rétrospectivement par des complétions d'éléments syntaxiques obligatoires, des compléments plutôt facultatifs ou des nouvelles UCT qui sont liées aux précédentes à l'aide de connecteurs. Notamment ces derniers cas soulignent l'importance et la fonctionnalisation de certaines ressources et, plus particulièrement, de certains lexèmes comme outil de gestion de l'alternance des locuteurs (ex. 1 à 4). De plus, un locuteur a le choix de traiter une UCT comme étant à un moment complète, tout en revendiquant son tour par le segment suivant (ex. 5). Une double orientation aussi bien vers une suite que vers une suspension du tour actuel est visible dans un formatage particulier de la fin du premier segment (p. ex. l'utilisation de lexèmes tels que "hein", en combinaison avec des allongements, ex. 7 \& 8). Il conviendrait alors de vérifier l'éventuelle récurrence (de groupements) de certaines ressources à des endroits systématiques de la CD afin de décrire de façon détaillée les variations de ce procédé en interaction française.

Le continuum sur lequel se trouvent les différents exemples de CD (entre compétition pour le tour et collaboration, entre complétude et incomplétude des segments) témoigne du caractère situé et négocié de la syntaxe en interaction (Auer 2005). Plutôt qu'une simple négociation de la complétude syntaxique, les séquences de $\mathrm{CD}$ montrent différents formatages pour négocier la complétude du droit à la parole ou bien l'acceptation momentanée d'un partage de ce droit (Szczepek 2000: 31). Une étude approfondie de ce phénomène interactionnel peut contribuer de manière significative à une compréhension de la syntaxe (et d'autres ressources grammaticales) comme étant une ressource exploitée pour la gestion de l'alternance des tours de parole et pour la négociation des droits et obligations des locuteurs moment par moment. 
Conventions de transcription

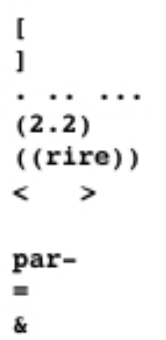

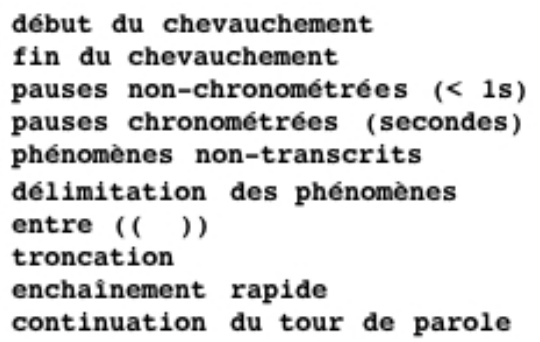

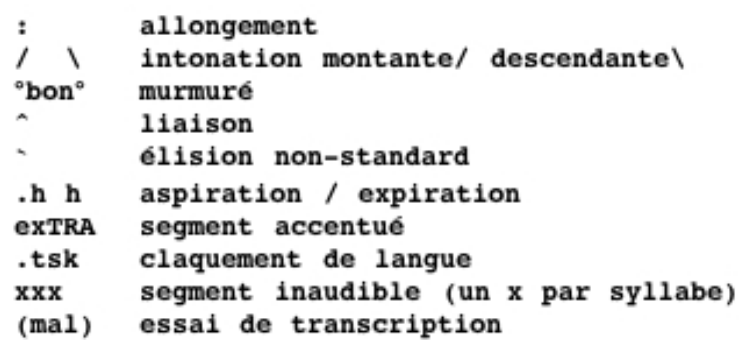

\section{Références bibliographiques}

Auer, P. (1996). The pre-front field in spoken German and its relevance as a grammaticalization position. Pragmatics, 6, 295-322.

Auer, P. (2002). Projection in Interaction and Projection in Grammar. InLiSt (Interaction and Linguistic Structures), 33 (www.uni-potsdam.de/u/inlist/).

Auer, P. (2005). Syntax als Prozess. InLiSt (Interaction and Linguistic Structures), 41 (www.unipotsdam.de/u/inlist/).

Auer, P., Günthner, S. (2003). Die Entstehung von Diskursmarkern im Deutschen - ein Fall von Grammatikalisierung? InLiSt (Interaction and Linguistic Structures), 38 (www.uni-potsdam.de/u/inlist/).

Bolden, G. B. (2003). Multiple modalities in collaborative turn sequences. Gesture, 3, 187-212.

Couper-Kuhlen, E., Ono, T. (2007). 'Incrementing' in conversation. A comparison of practices in English, German and Japanese. Pragmatics, 17 (Special Issue "Turn continuation in cross-linguistic perspective"), 513-552.

Ford, C. E., Fox, B. A., Thompson, S. A. (1996a). Interactional units in conversation: Syntactic, intonational, and pragmatic resources for turn management. Dans Ochs, E., Schegloff, E. A., Thompson, S. A. (éds), Interaction and Grammar. Cambridge: Cambridge University Press, 134-184.

Ford, C. E., Fox, B. A., Thompson, S. A. (1996b). Practices in the construction of turns: The 'TCU' revisited. Pragmatics, 6, 427-454.

Ford, C. E., Fox, B. A., Thompson, S. A. (2002). Constituency and the Grammar of Turn Increments. Dans Ford, C. E., Fox, B. A., Thompson, S. A. (éds.), The Language of Turn and Sequence. New York: Oxford University Press, 14 - 38.

Fox, B. A. (2007). Principles shaping grammatical practices: an exploration. Discourse Studies, 9, 299-318.

French, P., Local, J. (1986). Prosodic features and the management of interruptions. Dans Johns-Lewis, C. (éd.), Intonation in Discourse. London: Croom Helm, 157-180.

Goodwin, C. (1980). Restarts, Pauses, and the Achievement of a State of Mutual Gaze at Turn-Beginning. Sociological Inquiry, 50, 272-302.

Gülich, E., Mondada, L. (2001). Analyse conversationnelle. Dans Holtus, G., Metzelin M., Schmitt, C. (éds.), Lexikon der romanistischen Linguistik. Tübingen: Niemeyer, 196-250.

Heritage, J. (1984). A change-of-state token and aspects of its sequential placement. Dans Atkinson, J. M., Heritage, J. (éds.), Structures of social action. Studies in Conversation Analysis. Cambridge: Cambridge University Press, 299-345.

Jefferson, G. (1984). Notes on some orderliness of overlap onset. Dans D'Urso, V., Leonardi, P. (éds), Discourse Analysis and Natural Rhetorics. Cleup editore: Padova, 11-38.

Jefferson, G. (2004). A sketch of some orderly aspects of overlap in natural conversation. Dans Lerner, G. H. (éd.). Conversation Analysis. Studies from the first generation. Amsterdam: John Benjamins, 43-59.

Lerner, G. H. (1989). Notes on Overlap Managment in Conversation: The Case of Delayed Completion. Western Journal of Speech Communication, 53, 167-177. 
Lerner, G. H. (1991). On the syntax of sentences-in-progress. Language in Society, 20, 441-458.

Lerner, G. H. (2004). Collaborative Turn sequences. Dans Lerner, G. H. (éd.), Conversation Analysis. Studies from the first generation. Amsterdam: John Benjamins, 225-256.

Local, J., Kelly, J. (2006). Projection and 'Silences': Notes on Phonetic and Conversational Structure. Dans Drew, P., Heritage, J. (éds.), Conversation Analysis (vol. 3). London: Sage, 233-252.

Mondada, L. (1999). L'organisation séquentielle des ressources linguistiques dans l'élaboration collective des descriptions. Langage et société, 99, 9-36.

Mondada, L. (2004). Temporalité, séquentialité et multimodalité au fondement de l'organisation de l'interaction: Le pointage comme pratique de prise de tour. Cahiers de Linguistique française, 26, 269-292.

Mondada, L. (à paraître). L'interprétation online par les co-participants de la structuration du tour in fieri en TCUs: évidences multimodales. Travaux neuchâtelois de linguistique.

Mondada, L., Oloff, F. (à paraître). Overlap and multimodality: Rethinking overlap through video analysis. Dans Filliettaz, L.; Norris, S. (éds.), Multimodal Discourse in Practice. Oxford: Routledge.

Pomerantz, A. (1984). Agreeing and Disagreeing with Assessments: Some Features of Preferred / Dispreferred Turn Shapes. Dans Atkinson, J. M., Heritage, J. (éds), Structures of Social Action: Studies in Conversation Analysis. Cambridge: Cambridge University Press, 57-101.

Sacks, H. (1992). Lectures on Conversation. Oxford: Basil Blackwell.

Sacks, H., Schegloff, E. A., Jefferson, G. (1974). A simplest systematics for the organisation of turn taking for conversation. Language, 50, 696-735.

Schegloff, E. A. (1987). Recycled Turn Beginnings: A Precise Repair Mechanism in Conversation's Turn-taking Organization. Dans Button, G., Lee, J. R. E. (éds.), Talk and Social Organization. Clevedon: Multilingual Matters, 70-85.

Schegloff, E. A. (2000). Overlapping talk and the organization of turn-taking for conversation. Language in Society, $29,1-63$

Schegloff, E. A. (2002). Accounts of Conduct in Interaction. Interruption, Overlap, and Turn-Taking. Dans Turner, J.H. (éd.), Handbook of Sociological Theory. New York: Kluwer Academic / Plenum Publishers, 287-321.

Selting, M. (2000). The construction of units in conversational talk. Language in Society, 29, 477-517.

ten Have, P. (1999). Doing Conversation Analysis. A Practical Guide. Sage: London.

Szczepek, B. (2000). Formal Aspects of Collaborative Productions in English Conversation. InLiSt (Interaction and Linguistic Structures ), 17 (www.uni-potsdam.de/u/inlist/).

Vorreiter, S. (2003). Turn Continuations: Towards a Cross-Linguistic Classification. InLiSt (Interaction and Linguistic Structures), 39 (www.uni-potsdam.de/u/inlist/). 